Published in final edited form as:

South Med J. 2018 December ; 111(12): 746-753. doi:10.14423/SMJ.0000000000000905.

\title{
Invasive Mechanical Ventilation
}

\author{
James M. Walter, MD, Thomas C. Corbridge, MD, and Benjamin D. Singer, MD \\ Department of Medicine, Division of Pulmonary and Critical Care Medicine, Northwestern \\ University Feinberg School of Medicine, Chicago, Illinois
}

\begin{abstract}
Invasive mechanical ventilation is a potentially lifesaving intervention for acutely ill patients. The goal of this review was to provide a concise, clinically focused overview of basic invasive mechanical ventilation for the many clinicians who care for mechanically ventilated patients. Attention is given to how common ventilator modes differ in delivering a mechanical breath, evaluation of respiratory system mechanics, how to approach acute changes in airway pressure, and the diagnosis of auto-positive end-expiratory pressure. Waveform interpretation is emphasized throughout the review.
\end{abstract}

\section{Keywords}

mechanical ventilation; respiratory mechanics

Invasive mechanical ventilation is a lifesaving tool commonly used in the care of hospitalized patients. Because of a variety of factors, including an aging population, the number of patients who receive mechanical ventilation is increasing. ${ }^{1,2}$ Despite the increasing prevalence of invasive mechanical ventilation, providers in a variety of care settings report inadequate education on the use of mechanical ventilation. 3,4

\section{Components of Invasive Mechanical Ventilation}

Invasive mechanical ventilation includes an endotracheal tube (ETT) and a mechanical ventilator (as opposed to noninvasive ventilation in which the interface is a face mask). In addition to serving as the conduit for delivery of mechanical breaths, the ETT protects the airway, allows for suctioning of secretions, and facilitates select procedures, including bronchoscopy. Invasive mechanical ventilation helps stabilize patients with hypoxemic and hypercapnic respiratory failure, decreases inspiratory work of breathing, redistributes blood flow from exercising respiratory muscles to other tissues in patients with shock, and allows for the implementation of lung-protective (low tidal volume) ventilation in patients with acute respiratory distress syndrome (ARDS)..$^{5-7}$

Correspondence to Dr James M. Walter, Department of Medicine, Division of Pulmonary and Critical Care, Northwestern University Feinberg School of Medicine, 240 E Huron, McGaw M-300, Chicago, IL 60611. james.walter@ northwestern.edu.

J.M.W. has received compensation from the Lung Sciences Training Program, Northwestern University. T.C.C. has received compensation from the American Association of Nurse Practitioners and Wilson, Elser, Moskowitz, Edelman, and Dicker, Chicago. B.D.S. has received compensation from the National Institutes of Health and the Parker B. Francis Research Opportunity Award.

APlease define Pi. 


\section{Key Terms}

Ventilators are inspiratory assist devices that integrate volume, pressure, time, and flow (each as dependent or independent variables) to deliver a tidal breath under positive pressure. How the clinician sets these variables determines the mode. If a tidal volume is set and the pressure that results from delivering that volume is not, then the patient is receiving volume-controlled (VC) ventilation. Conversely, if a pressure is set and the tidal volume is not, then the patient is receiving pressure-controlled (PC) ventilation. Within VC there are two common strategies of breath sequencing: assist control (AC) and synchronized intermittent mandatory ventilation (SIMV). In PC, we discuss AC, pressure support (PS), SIMV, and pressure-regulated volume controlled (PRVC). A discussion of advanced modes of ventilation including airway pressure release ventilation and BiLevel are beyond the scope of this article, but have been reviewed elsewhere. ${ }^{8}$

To understand differences among available modes, it is important to be familiar with three terms: trigger, target, and cycle. "Trigger" refers to the stimulus that initiates a breath. Breaths initiated by a patient's effort are patient triggered. The ventilator senses effort through changes in airway pressure (pressure triggered) or inspiratory flow (flow triggered). The pressure or flow threshold required to trigger a breath is adjustable and termed the trigger sensitivity. Alternatively, breaths can be time triggered if the patient does not initiate a breath in the time required to achieve a set respiratory rate (RR). For example, if the RR is set at 12 breaths per minute, absent patient efforts, the ventilator delivers a breath every 5 seconds to ensure that the target RR is achieved. "Target" refers to the breath delivery strategy - a parameter that is set, achieved, and maintained throughout inspiration. This can be a set inspiratory flow rate and pattern or pressure, depending on the mode. "Cycle" describes how the breath is terminated. This can be a delivered tidal volume $\left(\mathrm{V}_{\mathrm{T}}\right)$, the end of a set inspiratory time $\left(\mathrm{T}_{\mathrm{i}}\right)$, or a change in inspiratory flow rate.

\section{AC}

The most common mode of mechanical ventilation in intensive care units is the AC mode (also referred to as continuous mandatory/mechanical ventilation on some models). ${ }^{9}$ A key feature of $\mathrm{AC}$ is that the patient receives a supported breath with both patient-triggered (assisted) and time-triggered (controlled) breaths. Positive end-expiratory pressure (PEEP), $\mathrm{RR}$, and the fraction of inspired oxygen $\left(\mathrm{FiO}_{2}\right)$ also are set. $\mathrm{AC}$ is available in VC and PC.

\section{AC-VC}

In $\mathrm{AC}-\mathrm{VC}$, breaths may be patient triggered or time triggered. The patient triggers the breath by generating a requisite change in airway pressure or inspiratory flow. Patients receive a time-triggered breath if they do not trigger a breath in the time required to meet the set RR. $\mathrm{AC}-\mathrm{VC}$ is flow targeted, as a set flow rate and pattern drive breath delivery. Both the flow rate in liters per minute (LPM) and the flow pattern (either a square/constant flow or a ramp/ decelerating flow) are set. It is important to recognize that in AC-VC, the patient receives the set flow rate and pattern with every breath. Clinicians should choose settings that approximate the patient's needs and optimize patient-ventilator synchrony. In general, decelerating waveforms are better tolerated and are the default choice by many intensivists. A square/constant waveform reduces inspiratory time and thus often is used to help prolong 
expiratory time in patients at risk for gas trapping and auto-PEEP (eg, patients with severe asthma). ${ }^{10}$ With a constant flow, the tidal volume is delivered more rapidly than with a decelerating waveform, thus maximizing the time a patient is able to exhale. Recommended average flow rates range between 30 and $60 \mathrm{LPM}$. With a square flow pattern, flow is constant. With a ramp pattern, the initial flow rate is set and the machine linearly decelerates flow to near zero during breath delivery. As such, the average flow rate in a patient set to receive 80 LPM by a ramp pattern is approximately 40 LPM on most ventilators.

Breaths in AC-VC are volume cycled. Inspiratory flow continues until a set $\mathrm{V}_{\mathrm{T}}$ is achieved, after which an exhalation valve opens and airway pressure drops to the set PEEP. AC-VC is therefore patient or time triggered, flow targeted, and volume cycled (Fig. 1A).

In AC-VC, airway pressure is not under direct control of the clinician but is rather a function of the compliance and resistance of the respiratory system and patient effort. When changing a patient's $\mathrm{V}_{\mathrm{T}}$ or flow settings, attention should be paid to how these changes affect airway pressure, in particular the pressure obtained during an inspiratory hold maneuver termed the plateau pressure $\left(\mathrm{P}_{\mathrm{plt}}\right)$, which measures the distending pressure of the respiratory system (discussed further below). Maintaining a $\mathrm{P}_{\text {plt }}<30 \mathrm{~cm} \mathrm{H}_{2} \mathrm{O}$ has been associated with improved mortality for patients with ARDS and should be closely monitored for all patients on mechanical ventilation. ${ }^{5}$ For patients ventilated in AC-VC, changes in clinical status (eg, worsening ARDS, pneumothorax) will be reflected by changes in airway pressure as $\mathrm{V}_{\mathrm{T}}$ remains fixed with each breath.

\section{AC-PC}

As in $\mathrm{AC}-\mathrm{VC}$, breaths in AC-PC may be patient or time triggered. Inspiration in AC-PC is pressure targeted. The clinician chooses an inspiratory pressure (also known as the driving pressure) to be given above PEEP and maintained throughout inspiration. These pressures are additive so that the peak pressure maintained during breath delivery is the sum of the set PEEP and set inspiratory pressure (eg, a PEEP of $5 \mathrm{~cm} \mathrm{H}_{2} \mathrm{O}$ and an inspiratory pressure of $15 \mathrm{~cm} \mathrm{H} \mathrm{H}_{2} \mathrm{O}$ will result in a constant peak pressure of $20 \mathrm{~cm} \mathrm{H}_{2} \mathrm{O}$ during breath delivery). As in $\mathrm{AC}-\mathrm{VC}$, clinicians should aim to achieve a $\mathrm{P}_{\text {plt }}$ measured during an end-inspiratory hold of $<30 \mathrm{~cm} \mathrm{H}_{2} \mathrm{O}$ to reduce the risk of barotrauma. In contrast to AC-VC, in which the clinician chooses the flow rate and pattern, in AC-PC the ventilator delivers flow in a decelerating pattern to achieve the preset constant pressure.

AC-PC is time cycled. The preset inspiratory pressure is provided for a set $\mathrm{T}_{\mathrm{i}}$, after which the exhalation valve opens and airway pressure returns to PEEP. AC-PC is therefore patient or time triggered, pressure targeted, and time cycled (Fig. 1B). Because $T_{i}$ can be directly adjusted, the clinician can easily increase the time that a patient spends in inspiration. Inverse ratio ventilation refers to a strategy in which the ratio of inspiratory time to expiratory time exceeds 1 . Because mean airway pressure is directly proportional to inspiratory time, inverse ratio ventilation has been proposed as a way to improve alveolar recruitment and oxygenation for patients with ARDS. Data supporting the superiority of this strategy are lacking, however, and there is concern that it may increase the risk of barotrauma. ${ }^{11}$ 
Unlike in $\mathrm{AC}-\mathrm{VC}$ in which $\mathrm{V}_{\mathrm{T}}$ is set by the clinician, in $\mathrm{AC}-\mathrm{PC} \mathrm{V}_{\mathrm{T}}$ is determined by the patient's respiratory system mechanics, effort, the set inspiratory pressure, and the set $\mathrm{T}_{\mathrm{i}}$. Tidal volume should therefore be monitored after changes in ventilator settings. Similarly, as a set inspiratory pressure is achieved with every breath, changes in a patient's lung mechanics will be primarily reflected by changes in $\mathrm{V}_{\mathrm{T}}$.

\section{PRVC}

Many ventilators include an $\mathrm{AC}$ mode termed PRVC, also known as $\mathrm{VC}^{+}$or adaptive pressure ventilation on some models. In this mode, $\mathrm{T}_{\mathrm{i}}$ and a goal $\mathrm{V}_{\mathrm{T}}$ are set by the clinician. The ventilator delivers a constant inspiratory pressure with decelerating flow for the duration of the $\mathrm{T}_{\mathrm{i}}$, after which the breath is cycled off. This mode is therefore patient or time triggered, pressure targeted, and time cycled as in AC-PC (Fig. 1C). The unique aspect of PRVC is that the ventilator adjusts inspiratory pressure to correct discrepancies between the delivered and goal $\mathrm{V}_{\mathrm{T}}$. For example, if the set $\mathrm{V}_{\mathrm{T}}$ is $500 \mathrm{~mL}$ and the actual delivered $\mathrm{V}_{\mathrm{T}}$ for a given breath is $600 \mathrm{~mL}$, the ventilator will decrease inspiratory pressure on subsequent breaths to bring the achieved $\mathrm{V}_{\mathrm{T}}$ closer to the goal $\mathrm{V}_{\mathrm{T}}$.

Because flow is neither set by the clinician nor fixed, PRVC may improve patient-ventilator synchrony and reduce the potential for inappropriately low flow settings. Conversely, although a goal $\mathrm{V}_{\mathrm{T}}$ is set by the clinician, $\mathrm{V}_{\mathrm{T}}$ will fluctuate based on changes in effort and mechanics. As such, PRVC may decrease adherence to a lung-protective ventilation strategy and should be used with caution in patients with ARDS. It also may increase the work of breathing because increasing patient efforts will be met with decreasing ventilator support to ensure that the goal $\mathrm{V}_{\mathrm{T}}$ is achieved. As such, this mode may be most appropriate for patients who are clinically improving and moving toward liberation from mechanical ventilation.

\section{VC vs PC}

Evidence does not support the superiority of one AC mode over another. ${ }^{12}$ In general, the lack of a fixed flow rate in AC-PC may improve patient-ventilator synchrony, but it is unclear whether this translates into improved outcomes. ${ }^{13}$

\section{PS}

PS is a spontaneous mode of ventilation used most commonly during spontaneous breathing trials. In PS, there is no set RR and all breaths are patient triggered. In the absence of a backup mode that takes over during apnea, an apneic patient will not receive any breaths if he or she is ventilated using PS. Once a breath is triggered, an inspiratory pressure above the set PEEP is maintained throughout inspiration. PS is therefore pressure targeted.

PS is flow cycled, as inspiration is cycled off by a drop in inspiratory flow. This variable can be adjusted by the clinician and is typically set as a percentage of peak inspiratory flow (eg, when inspiratory flow falls to $25 \%$ of peak inspiratory flow). PS is therefore patient triggered, pressure targeted, and flow cycled (Fig. 2A). 
SIMV is a frequently used mode of mechanical ventilation. ${ }^{9}$ In SIMV, supported (mandatory) ventilator breaths are given at a set rate. If a patient attempts to trigger a breath in a preset time interval before the next IMV breath, the machine will deliver a supported breath (the mandatory breaths are therefore synchronized to patient effort). Mandatory breaths can be delivered with a VC (flow targeted, volume cycled), PC (pressure targeted, time cycled), or PRVC (pressure targeted, time cycled) strategy as in the AC mode.

The key difference between SIMV and AC is that patients ventilated in SIMV may take unassisted spontaneous breaths between mandatory machine breaths (Fig. 2B). Often, a small amount of pressure support is added to these breaths to overcome the resistive load of the ETT and augment $\mathrm{V}_{\mathrm{T}}$. The $\mathrm{V}_{\mathrm{T}}$ achieved with these breaths is not controlled by the clinician but rather a function of patient effort and respiratory system mechanics. Use of SIMV in a spontaneously breathing patient often results in two unique ventilator waveforms: supported mandatory breaths and spontaneous pressure-supported breaths. In patients without spontaneous efforts (eg, because of neuromuscular blockade), SIMV is identical to $\mathrm{AC}$, as the patient will receive only time-triggered machine breaths.

SIMV has been purported to reduce respiratory muscle disuse atrophy, improve ventilator synchrony, and prevent respiratory alkalosis ${ }^{14}$; however, in patients whose spontaneous RR is significantly higher than the set IMV rate, SIMV may promote respiratory muscle fatigue. ${ }^{15}$ Although there is a lack of convincing evidence to support or discourage the use of SIMV as the primary mode of mechanical ventilation in acutely ill patients, several well-conducted trials have found SIMV inferior to other methods during ventilator weaning. ${ }^{16,17}$

\section{Ventilator Settings: Getting Started}

Initial ventilator settings for patients placed on invasive mechanical ventilation should be guided by the cause of respiratory failure, the goals of mechanical ventilation, and the patient's comorbidities. In general, ventilation is manipulated by changes in $\mathrm{V}_{\mathrm{T}}$ and $\mathrm{RR}$. To improve oxygenation, $\mathrm{FiO}_{2}$ and/or PEEP can be increased. PEEP improves oxygenation by recruiting collapsed alveoli and decreasing intrapulmonary shunt. ${ }^{18}$ Below, we provide an overview of ventilator strategies for three common scenarios encountered in clinical practice.

\section{Normal Minute Ventilation}

Some patients who require mechanical ventilation will have a normal minute ventilation $(\approx 6-8 \mathrm{~L} / \mathrm{min}$ ). These include patients intubated for upper airway obstruction (eg, angioedema), altered mental status (eg, ethanol intoxication), and those undergoing surgery. In these cases, the following settings are likely to achieve an adequate $\mathrm{PaO}_{2}\left(\mathrm{eg}, \mathrm{PaO}_{2} 60-80\right.$ $\mathrm{mm} \mathrm{Hg}, \mathrm{SpO}_{2}>88 \%$ ) and acceptable $\mathrm{PaCO}_{2}$ (eg, 30-50 mm Hg) in most adult patients:

- $\quad$ Mode: AC-VC

- $\quad$ RR: 14 breaths per minute

- $\quad \mathrm{V}_{\mathrm{T}}: 7$ to $8 \mathrm{~mL} / \mathrm{kg}$ ideal body weight (IBW, based on a patient's height and sex) 
- $\mathrm{FiO}_{2}: 0.4$ to 1.0 , depending on the clinical scenario

- $\quad$ PEEP: $5 \mathrm{~cm} \mathrm{H}_{2} \mathrm{O}$

- Inspiratory flow rate and pattern: 80 LPM using a decelerating/ramp flow

Alternatively, in patients with normal lungs and an intact mental status, PS can be used. Reasonable initial settings include a PEEP of $5 \mathrm{~cm} \mathrm{H}_{2} \mathrm{O}$, Pi of $15 \mathrm{~cm} \mathrm{H}_{2} \mathrm{O}$, and an $\mathrm{FiO}_{2}$ of 0.4. ${ }^{\mathrm{A}}$ Subsequent adjustments to Pi should target an RR of roughly 14 breaths per minute and a $\mathrm{V}_{\mathrm{T}}$ of 8 to $10 \mathrm{~mL} / \mathrm{kg}$ IBW.

\section{ARDS}

For patients with ARDS, a ventilator strategy that prioritizes low-tidal volumes and low plateau pressures, termed "lung-protective ventilation," has been shown to improve mortality. ${ }^{5}$ Reasonable initial settings for a patient with ARDS include the following:

- $\quad$ Mode: AC-VC

- $\quad$ RR: 20 breaths per minute

- $\mathrm{V}_{\mathrm{T}}: 7$ to $8 \mathrm{~mL} / \mathrm{kg} \mathrm{IBW}$

- $\quad \mathrm{FiO}_{2}: 1.0$

- $\quad$ PEEP: $5 \mathrm{~cm} \mathrm{H}_{2} \mathrm{O}$

- Inspiratory flow rate and pattern: 80 LPM using a decelerating/ramp flow

Note the higher initial RR that is needed to match the high minute ventilation of patients with ARDS. The $\mathrm{V}_{\mathrm{T}}$ should be decreased over several hours to a goal of $6 \mathrm{~mL} / \mathrm{kg}$ IBW. The $\mathrm{RR}$ is increased in parallel with the decrease in $\mathrm{V}_{\mathrm{T}}$ to maintain an adequate minute ventilation and avoid progressive hypercapnia and acidemia. Tidal volume should be decreased further if necessary to achieve a $\mathrm{P}_{\text {plt }}<30 \mathrm{~cm} \mathrm{H}_{2} \mathrm{O}$. PEEP and $\mathrm{FiO}_{2}$ are adjusted in stepwise fashion to maintain a $\mathrm{PaO}_{2}$ of $55-80 \mathrm{~mm} \mathrm{Hg}{ }^{5}$

\section{Severe Obstructive Lung Disease}

For patients with status asthmaticus or chronic obstructive pulmonary disease, ventilation should allow for complete exhalation to prevent the development of auto-PEEP (discussed further below). This is most effectively accomplished by limiting $\mathrm{RR}$ and $\mathrm{V}_{\mathrm{T}} \cdot{ }^{10}$ Reasonable initial settings include the following:

- $\quad$ Mode: AC-VC

- $\quad$ RR: 10 to 14 breaths per minute

- $\quad \mathrm{V}_{\mathrm{T}}: 7$ to $8 \mathrm{~mL} / \mathrm{kg} \mathrm{IBW}$

- $\quad \mathrm{FiO}_{2}: 1.0$

- $\quad$ PEEP: $5 \mathrm{~cm} \mathrm{H}_{2} \mathrm{O}$

- Inspiratory flow rate and pattern: 60 LPM using a square waveform 
For patients with severe bronchospasm, clinicians should target a minute ventilation of 6 to 8 $\mathrm{L} /$ minute to prevent auto-PEEP. Deep sedation and neuromuscular blocking agents may be required to achieve this goal. ${ }^{10}$ Elevations in $\mathrm{PaCO}_{2}$ are tolerated to facilitate the reduction in minute ventilation provided the $\mathrm{pH}$ does not drop below 7.15. The set flow rate can be increased above 60 LPM to further shorten inspiratory time, although this strategy provides only marginal additional benefit when minute ventilation is low. High flow rates will increase peak pressure because of an increase in airway resistive pressure. Because these changes do not increase the distending pressure of the lung (as measured by $\mathrm{P}_{\mathrm{plt}}$ ), isolated elevations in peak pressures are not necessarily harmful.

\section{Respiratory System Mechanics}

Once a patient is placed on invasive mechanical ventilation, the ventilator can be used to measure plateau pressure, airway resistance, and the static compliance of the respiratory system, collectively referred to as respiratory system mechanics. Knowledge of how to obtain and interpret these values is essential for optimal care. ${ }^{19}$

\section{Equation of Motion}

The equation of motion relates the pressure at the airway opening (Pao) to lung volume, flow, respiratory system mechanics, and patient effort, as follows:

$$
\text { Pao }=\frac{V}{C s t r s}+(\dot{V} \times R)-\text { Pmus }
$$

where $\mathrm{V}$ is lung volume above functional residual capacity, Cstrs is the static compliance of the respiratory system, $\dot{V}$ is inspiratory flow rate, $\mathrm{R}$ is inspiratory resistance, and Pmus is the pressure generated by the patient's respiratory muscles. The product $\dot{V} \times R$ reflects the pressure required to overcome the frictional forces generated by flow through the ETT and airways (otherwise known as resistive pressure). Under zero flow conditions $(\dot{V} \times R=0)$, in a passive patient without spontaneous respiratory effort (Pmus $=0$ ), the distending pressure of the respiratory system or $\mathrm{P}_{\text {plt }}$ is determined by $\mathrm{V}_{\mathrm{T}}$ and Cstrs, as follows:

$$
P p l t=\frac{V t}{C s t r s}+P E E P
$$

The equation of motion predicts that as distending pressure increases with a tidal breath, resistive pressure must decrease to maintain a constant inspiratory pressure. Constant pressure waveforms therefore mandate a decelerating inspiratory flow pattern (Fig. 3A). Conversely, use of a constant flow pattern (where resistive pressure remains constant during a tidal breath) produces a steady rise in airway pressure with lung inflation (visualized as a "shark fin" pressure waveform).

\section{Resistance and Compliance}

Pressures generated during breath delivery are determined by the compliance of the respiratory system and airway resistance. It is not possible to determine the respective 
contributions of each of these components without performing an inspiratory hold maneuver (Fig. 3B). A valid inspiratory hold maneuver requires minimal or no effort on the part of the patient.

Compliance measurements do not depend upon the flow delivery strategy because they are obtained under zero flow conditions. Because compliance is the differential change in volume for a given change in pressure $\left(\frac{\Delta V}{\Delta P}\right)$, the Cstrs is calculated by

$$
C_{\text {strs }}=\frac{V_{T}}{P_{\text {plat }}-P E E P_{\text {total }}}
$$

Normal compliance in a ventilated patient is 60 to $80 \mathrm{~mL} / \mathrm{cm} \mathrm{H}_{2} \mathrm{O}$. Causes of decreased compliance include pulmonary edema, interstitial lung disease, auto-PEEP, pleural disease, chest wall deformity, obesity, and ascites. In patients with low Cstrs, $\mathrm{P}_{\mathrm{plt}}$ is higher for any given $\mathrm{V}_{\mathrm{T}}$.

Resistance is pressure divided by flow. As such, airway resistance is calculated by the following:

$$
R_{a w}=\frac{P I P-P_{p l a t}}{\text { flow }}
$$

Normal airway resistance on a mechanical ventilator is $<15 \mathrm{~cm} \mathrm{H}_{2} \mathrm{O} / \mathrm{L} / \mathrm{second}$, assuming a normal-sized ETT. If the patient is receiving a decelerating flow pattern, this should be switched to constant flow (at $60 \mathrm{LPM}$, which is $1 \mathrm{~L} / \mathrm{s}$, thus making the calculation simpler) to obtain valid resistance measurements. Increased airway resistance suggests a kinked or plugged endotracheal tube, intraluminal mucus, or bronchospasm. Many ventilators will calculate and display airway resistance and compliance when an inspiratory hold is performed.

\section{Evaluating Changes in Airway Pressure}

A frequent diagnostic challenge when caring for mechanically ventilated patients is responding to sudden changes in airway pressure. Assessing respiratory system compliance and airway resistance facilitates the development of a focused differential diagnosis and helps guide initial management (Fig. 3C). Sudden changes in airway pressure that result in hemodynamic instability should be managed by disconnecting the patient from the mechanical ventilator and ventilating them with a bag valve mask device connected to the ETT at a rate of 8 to 10 breaths per minute. Outside this scenario, the initial diagnostic step should be to perform an inspiratory hold maneuver. Increased peak airway pressure coupled with an elevated $\mathrm{P}_{\mathrm{plt}}$ suggests decreased lung compliance. When this change occurs acutely, pneumothorax and auto-PEEP should be rapidly excluded.

An increase in peak pressure with a low $\mathrm{P}_{\text {plt }}$ suggests increased large-airway resistance. The ETT should be examined to ensure that it is not kinked or occluded by the patient's teeth, 
tube patency should be confirmed with the passage of a suction catheter, and the patient's chest should be auscultated for evidence of bronchospasm.

Sudden decreases in airway pressure suggest that air is escaping from the ventilator circuit. This can be caused by a defect in the ETT, a malfunctioning ETT balloon, or a large air leak in the setting of chest tube drainage.

\section{Auto-PEEP}

As noted above, clinicians should monitor for the development of auto-PEEP, which is defined by an EEP above machine-set PEEP. ${ }^{20,21}$ Auto-PEEP results from incomplete exhalation of the delivered breath, which leads to increased end-expiratory lung volumes. This is most commonly seen in conditions with increased airway resistance and reduced expiratory flow rates (eg, severe asthma, chronic obstructive pulmonary disease). The resulting increase in intrathoracic pressure decreases the gradient for venous return, increases right ventricular afterload, and increases the work of breathing because the patient must overcome this pressure to trigger a breath. In severe cases, these physiologic changes can be life threatening. ${ }^{10}$ Auto-PEEP can be estimated in several ways. Persistent expiratory flow at the start of inspiration (noted either by auscultation or observing an expiratory flow tracing that does not return to zero by the start of the next breath) suggests the presence of auto-PEEP (Fig. 4). An end-expiratory occlusion maneuver also can be performed whereby flow is stopped at the end of expiration and an occlusion pressure that approximates a patient's intrinsic PEEP is recorded. This measurement can only be reliably obtained in patients without spontaneous respiratory effort. Finally, in the absence of other known causes of decreased respiratory system compliance, an elevated $\mathrm{P}_{\mathrm{plt}}$ in an at-risk patient strongly suggests the presence of auto-PEEP. In addition to treating the underlying lung disease, strategies that prolong expiratory time such as decreased $R R$, low $V_{T}$, and increased inspiratory flow rates decrease auto-PEEP. If progressive auto-PEEP is believed to be the cause of hemodynamic instability, the patient should be disconnected from the ventilator and the chest manually decompressed.

\section{Conclusions}

Invasive mechanical ventilation is a powerful therapeutic and diagnostic tool in critically ill patients. Understanding modes and settings, measuring respiratory system mechanics, and paying attention to pressure and flow waveforms are essential components of delivering high-quality critical care.

\section{Acknowledgments}

This work was supported by Northwestern University's Lung Sciences Training Program (5T32HL076139-14), the National Institutes of Health (grant HL128867), and the Parker B. Francis Research Opportunity Award.

\section{References}

1. Carson SS, Cox CE, Holmes GM, et al. The changing epidemiology of mechanical ventilation: a population-based study. J Intensive Care Med 2006;21:173-182. [PubMed: 16672639] 
2. Halpern NA, Goldman DA, Tan KS, et al. Trends in critical care beds and use among population groups and Medicare and Medicaid beneficiaries in the United States: 2000-2010. Crit Care Med 2016;44:1490-1499. [PubMed: 27136721]

3. Cox CE, Carson SS, Ely EW, et al. Effectiveness of medical resident education in mechanical ventilation. Am J Respir Crit Care Med 2003;167:32-38. [PubMed: 12406827]

4. Wilcox SR, Strout TD, Schneider JI, et al. Academic emergency medicine physicians' knowledge of mechanical ventilation. West J Emerg Med 2016;17:271-279. [PubMed: 27330658]

5. Acute Respiratory Distress Syndrome Network, Brower RG, Matthay MA, et al. Ventilation with lower tidal volumes as compared with traditional tidal volumes for acute lung injury and the acute respiratory distress syndrome. N Engl J Med 2000;342:1301-1308. [PubMed: 10793162]

6. Cinnella G, Conti G, Lofaso F, et al. Effects of assisted ventilation on the work of breathing: volume-controlled versus pressure-controlled ventilation. Am J Respir Crit Care Med 1996;153:1025-1033. [PubMed: 8630541]

7. Kapitan KS. Ventilatory failure. Can you sustain what you need? Ann Am Thorac Soc 2013;10:396399. [PubMed: 23952866]

8. Singer BD, Corbridge TC. Pressure modes of invasive mechanical ventilation. South Med J 2011;104:701-709. [PubMed: 21941160]

9. Esteban A, Anzueto A, Alia I, et al. How is mechanical ventilation employed in the intensive care unit? An international utilization review. Am J Respir Crit Care Med 2000;161:1450-1458. [PubMed: 10806138]

10. Leatherman J Mechanical ventilation for severe asthma. Chest 2015;147:1671-1680. [PubMed: 26033128]

11. Shanholtz C, Brower R. Should inverse ratio ventilation be used in adult respiratory distress syndrome? Am J Respir Crit Care Med 1994;149:1354-1358. [PubMed: 8173778]

12. Chacko B, Peter JV, Tharyan P, et al. Pressure-controlled versus volume-controlled ventilation for acute respiratory failure due to acute lung injury (ALI) or acute respiratory distress syndrome (ARDS). Cochrane Database Syst Rev 2015;1:CD008807. [PubMed: 25586462]

13. MacIntyre NR, McConnell R, Cheng KC, et al. Patient-ventilator flow dyssynchrony: flow-limited versus pressure-limited breaths. Crit Care Med 1997;25:1671-1677. [PubMed: 9377881]

14. Kacmarek RM, Branson RD. Should intermittent mandatory ventilation be abolished? Respir Care 2016;61:854-866. [PubMed: 27235318]

15. Marini JJ, Smith TC, Lamb VJ. External work output and force generation during synchronized intermittent mechanical ventilation. Effect of machine assistance on breathing effort. Am Rev Respir Dis 1988;138:1169-1179. [PubMed: 3202477]

16. Brochard L, Rauss A, Benito S, et al. Comparison of three methods of gradual withdrawal from ventilatory support during weaning from mechanical ventilation. Am J Respir Crit Care Med 1994;150:896-903. [PubMed: 7921460]

17. Esteban A, Frutos F, Tobin MJ, et al. A comparison of four methods of weaning patients from mechanical ventilation. Spanish Lung Failure Collaborative Group. N Engl J Med 1995;332:345350. [PubMed: 7823995]

18. Sahetya SK, Goligher EC, Brower RG. Fifty years of research in ARDS. Setting positive endexpiratory pressure in acute respiratory distress syndrome. Am J Respir Crit Care Med 2017;195:1429-1438. [PubMed: 28146639]

19. Stansbury RC, Long JL, Petsonk EL. Respiratory mechanics in a patient requiring neuromuscular blockade. Ann Am Thorac Soc 2017;14:288-292. [PubMed: 28146382]

20. Tobin MJ, Lodato RF. PEEP, auto-PEEP, and waterfalls. Chest 1989;96:449-451. [PubMed: 2670461]

21. Marini JJ. Dynamic hyperinflation and auto-positive end-expiratory pressure: lessons learned over 30 years. Am J Respir Crit Care Med 2011;184:756-762. [PubMed: 21700908] 


\section{Key Points}

- Modes of ventilation can be described by three key variables: "trigger" (the stimulus that initiates a mechanical breath), "target" (the breath-delivery strategy), and "cycle" (how the breath is terminated).

- $\quad$ Measuring respiratory system mechanics facilitates the development of a differential diagnosis and helps guide initial management for mechanically ventilated patients who experience sudden changes in airway pressure. 

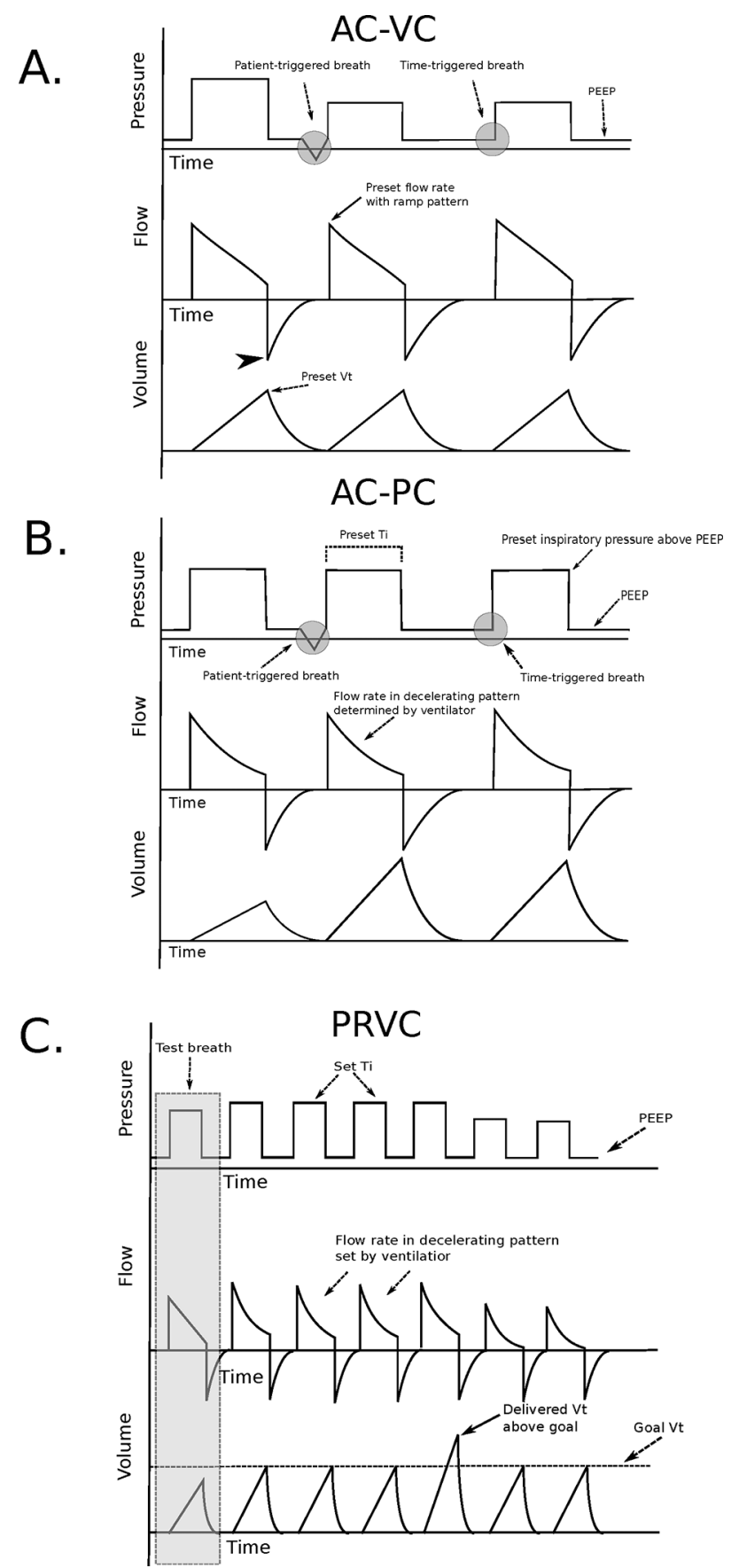

Fig. 1.

(A) Pressure, volume, and time waveforms in assist-control volume control (AC-VC) mode using a decelerating flow strategy. Note the second breath is patient triggered as evidenced by a negative deflection of the pressure waveform. The flow rate set on the ventilator represents the peak flow rate (solid black arrow), which linearly decelerates to near zero with each breath. Inspiration ends after a preset tidal volume is achieved. Peak airway pressure is not set by the clinician but rather a consequence of respiratory system mechanics, tidal volume, inspiratory flow rate and pattern, and patient effort. Note that during the 
second and third breaths, airway pressure decreases while flow rate and tidal volume remain unchanged reflecting a favorable change in respiratory system mechanics. Expiratory flow (arrowhead) is not controlled by the ventilator but is instead dependent on patient effort and respiratory system mechanics. $(B)$ Pressure, volume, and time waveforms in the assistcontrol pressure control (AC-PC) mode. Note that the second breath is patient triggered as evidenced by a negative deflection of the pressure waveform. With each breath, a preset inspiratory pressure above positive end-expiratory pressure is delivered for a set inspiratory time, after which the breath is cycled off. Tidal volume is determined by the patient's respiratory system mechanics, inspiratory effort, inspiratory pressure, and inspiratory time. Note that during the second and third breaths, tidal volume increases despite an unchanged inspiratory pressure and inspiratory time, reflecting a favorable change in respiratory system mechanics or increased patient effort. (C) Pressure, volume, and time waveforms in pressure-regulated volume control (PRVC) mode. During a test breath (gray box), the ventilator estimates the inspiratory pressure and flow rate needed to achieve a preset goal tidal volume in a preset inspiratory time. Inspiratory pressure is continually adjusted to correct discrepancies between the goal and delivered tidal volume. Note during the fifth breath the delivered tidal volume exceeds the goal tidal volume (solid arrow). Inspiratory pressure is decreased by the machine on subsequent breaths to bring the delivered tidal volume closer to the goal tidal volume. 

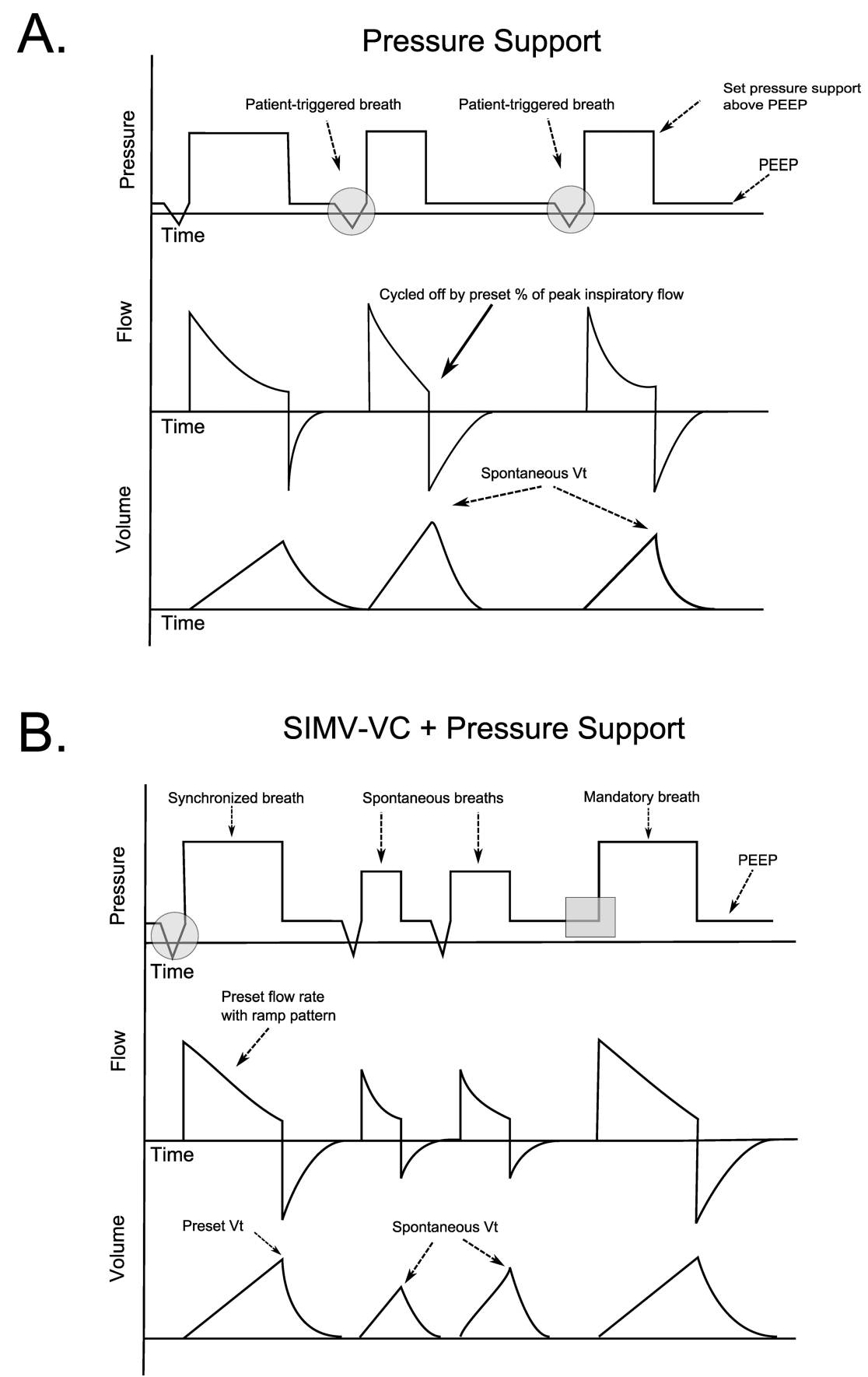

Fig. 2.

(A) Pressure, volume, and time waveforms in pressure support (PS) ventilation. All breaths are patient triggered as evidenced by the negative deflections in the pressure waveform (gray circles). With each breath, a set inspiratory pressure is delivered above positive endexpiratory pressure. Per the equation of motion, constant pressure waveforms require decelerating inspiratory flow, and when this inspiratory flow falls to a preset percentage of peak inspiratory flow (solid arrow), the machine cycles off. Tidal volume will vary based on respiratory system mechanics and patient effort. $(B)$ Pressure, volume, and time waveforms 
in SIMV-VC with pressure support. Flow-targeted volume-cycled mandatory breaths are given at a rate set by the clinician (gray box). These breaths are "synchronized" to patient effort (and thereby assisted) if a patient attempts to trigger a breath near the time of set breath delivery (gray circle). Between synchronized and mandatory breaths, the patient may take spontaneous breaths which are generally supported with pressure support (middle two breaths). 

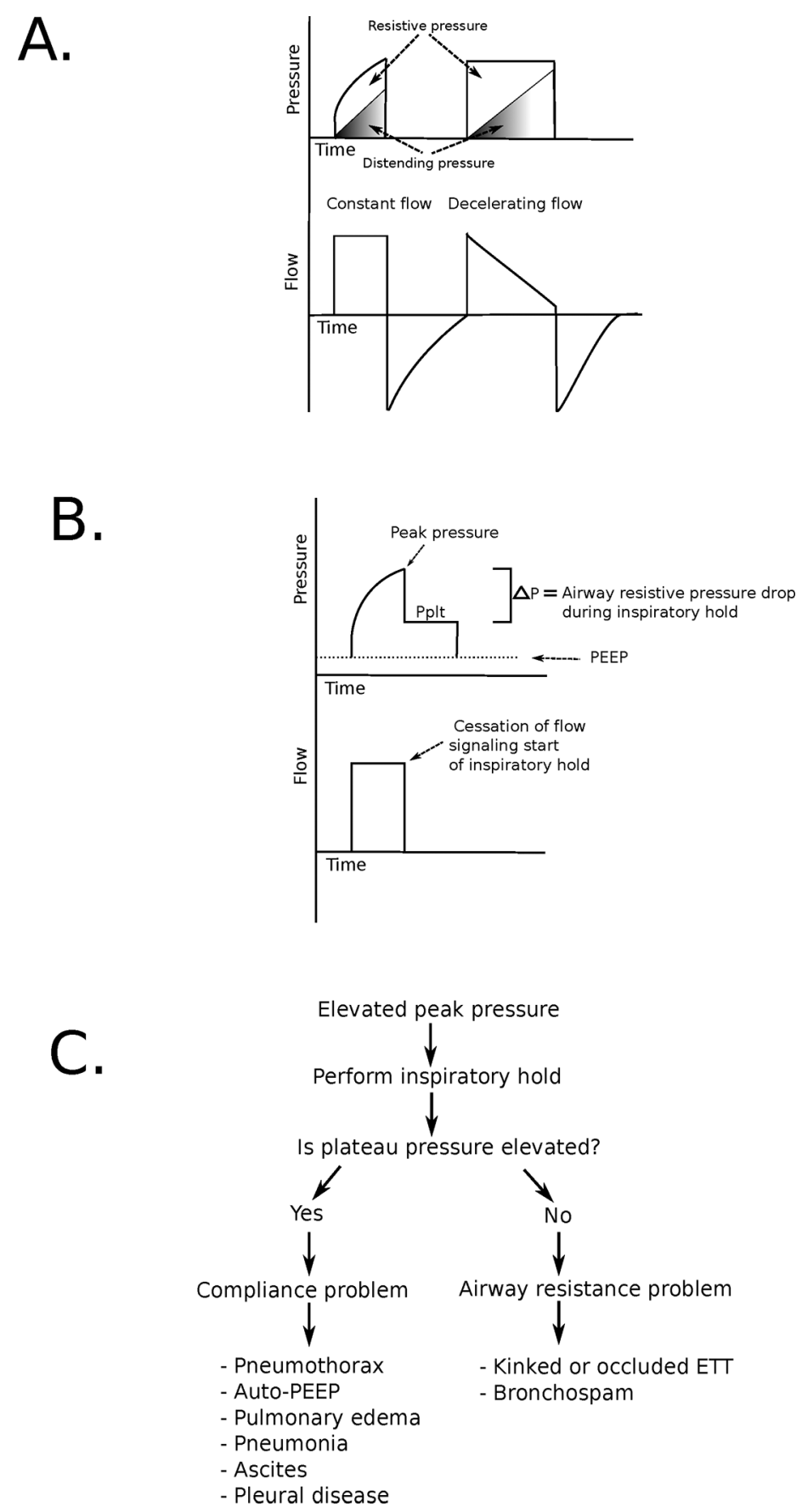

Fig. 3 .

$(A)$ Pressure and flow waveforms for constant (left) and decelerating (right) inspiratory flow patterns. Note the "shark fin" appearance of the pressure waveform with constant inspiratory flow caused by the steady increase in distending pressure (shaded area) coupled with a constant resistive pressure (unshaded area). With decelerating inspiratory flow, a square pressure waveform is observed as decreasing flow causes a decline in resistive pressure during breath delivery. $(B)$ Pressure and flow waveforms during an inspiratory hold maneuver. During inspiration, airway pressure rises from positive end-expiratory pressure 
(PEEP) to peak inspiratory pressure. Inspiratory flow is then stopped, eliminating resistive pressure and causing airway pressure to fall to a plateau pressure $\left(\mathrm{P}_{\mathrm{plt}}\right)$. The difference between peak inspiration pressure and $\mathrm{P}_{\mathrm{plt}}$ represents airway resistive pressure. The difference between $\mathrm{P}_{\mathrm{plt}}$ and PEEP is determined by tidal volume and respiratory system compliance. (C) Diagnostic approach to elevated peak pressures on a mechanical ventilator. ETT, endotracheal tube. 


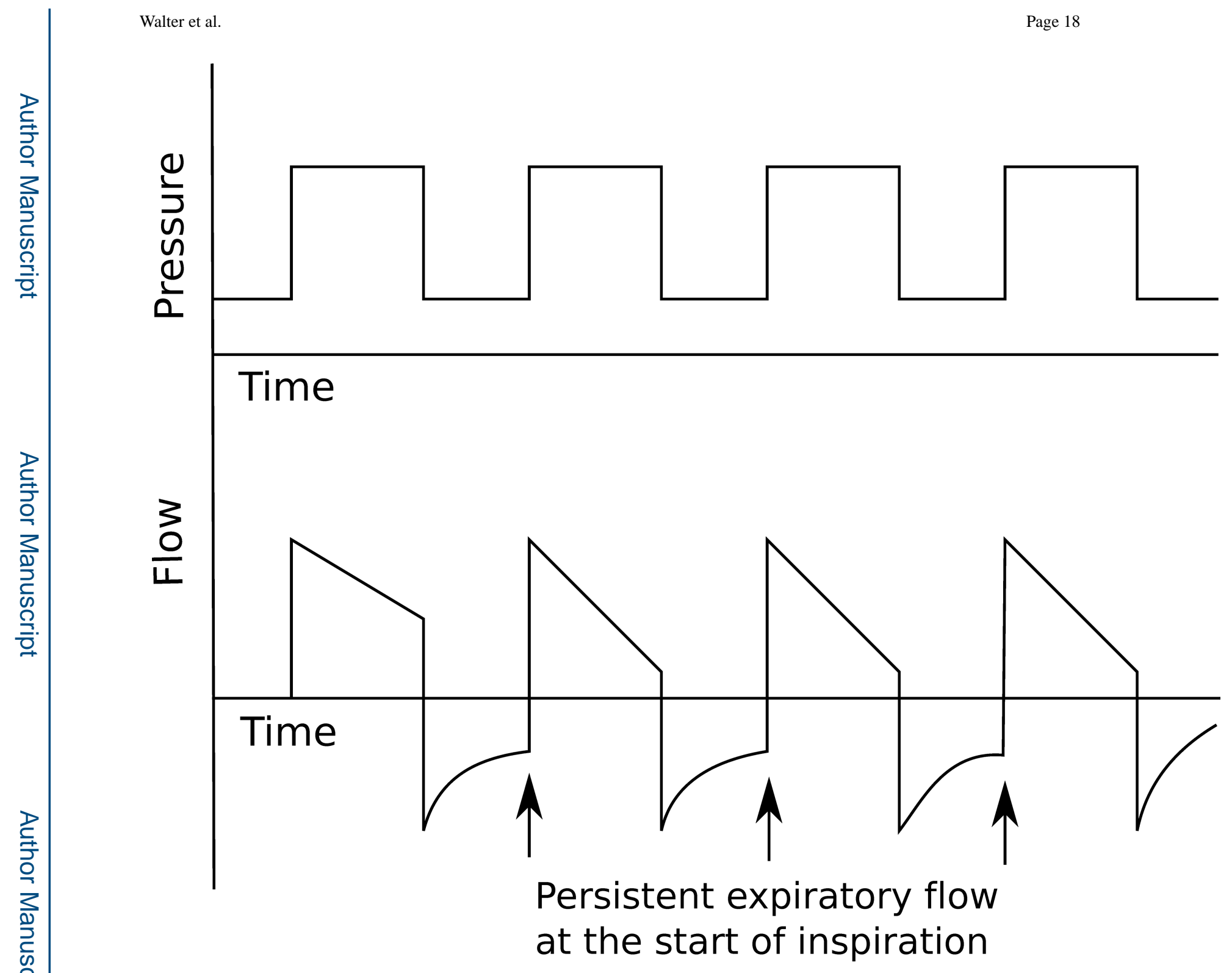

Fig. 4.

Pressure and flow waveforms in a patient with autopositive end-expiratory pressure. Note that expiratory flow does not return to zero before the start of the next breath (arrows). 